\title{
Estudio comparativo de la distancia coracohumeral en pacientes con patología degenerativa del subescapular y una población voluntaria asintomática
}

\section{Comparative Analysis of the Coracohumeral Distance between Patients with Degenerative Subscapularis Tendon Pathology and a Normal Age-matched Population}

\author{
Gabriel Cárdenas ${ }^{1,2}$ Christian Calderon ${ }^{3}$ Tomas Pineda ${ }^{4}$ Joaquin Avila ${ }^{5}$ Ricardo Pefaur ${ }^{6}$
}

\footnotetext{
${ }^{1}$ Unidad de Cirugía de Hombro, Departamento de Traumatología y Ortopedia, Instituto de Seguridad del Trabajo (IST), Viña del Mar, Chile

${ }^{2}$ Fellowship en Cirugía de Hombro, Institut Universitaire Locomoteur et du Sport (iULS), Hôpital Pasteur 2 CHU de Nice, France

3 Departamento de Imagenología Musculoesquelética, Instituto de

Seguridad del Trabajo (IST), Viña del Mar, Chile

${ }^{4}$ Residente de Traumatología y Ortopedia, Universidad de Chile, Santiago, Chile

${ }^{5}$ Interno de Medicina, Universidad de Valparaíso, Valparaíso, Chile

${ }^{6}$ Departamento de Bioestadística, Pontificia Universidad Católica de

Valparaíso, Chile
}

Rev Chil Ortop Traumatol 2019;60:9-15.

\author{
Address for correspondence Gabriel Cardenas, MD, Hôpital Pasteur \\ 2, 30 Voie Romaine, 06001 Nice, France \\ (e-mail: dr.gcardenaso@gmail.com).
}

\section{Resumen \\ Palabras Claves \\ - distancia coracohumeral \\ - pinzamiento subcoracoideo \\ - estenosis subcoracoidea \\ - subescapular \\ - coracoplastía}

Introducción El pinzamiento subcoracoideo secundario a una distancia coracohumeral ( $\mathrm{DCH}$ ) disminuida, ha sido descrito como una posible etiología de las lesiones degenerativas del tendón del Subescapular (SSC), sin embargo, esa teoría aún es controversial.

Objetivo Evaluar si existe una diferencia significativa entre la $\mathrm{DCH}$ promedio de pacientes con patología degenerativa del SSC y un grupo control.

Materiales y Métodos Se diseñó un estudio de casos-controles y se estableció un tamaño muestral mínimo de 36 casos por grupo. De nuestra base de datos, y dentro de un periodo de 6 meses, se rescataron 46 Resonancias Magnéticas (RM) de hombro con patología degenerativa avanzada y/o roturas del SSC (grupo SSC). El grupo control fue conformado por 36 RM realizadas a voluntarios asintomáticos sin patología del mango rotador. Se incluyeron en ambos grupos solo pacientes entre 40 y 60 años. Se utilizó prueba de t para determinar las diferencias entre 2 grupos con un nivel de confianza del $95 \%$.

Resultados La edad promedio del grupo SSC fue 50,1 $\pm 6,1$ años y del grupo control $51,7 \pm 6,8$ años, no existiendo diferencia estadísticamente significativa entre ambos $(p=0.43)$. La DCH promedio en el grupo SSC fue $8,58 \mathrm{~mm}$ [IC: 7,95-9,21 mm], y en el grupo control fue 11,04 mm [IC: 10,05-12,04 mm]. Al comparar la DCH, se encontró una diferencia estadísticamente significativa entre grupos $(p=0,00048)$. received

November 6, 2018 accepted

February 4, 2019

published online

March 8, 2019
DOI https://doi.org/

10.1055/s-0039-1683375. ISSN 0716-4548.
Copyright $(2019$ by Thieme Revinter

Publicações Ltda, Rio de Janeiro, Brazil
License terms

(c) $(1) \$$ 


\begin{abstract}
Keywords

- coracohumeral distance

- subcoracoid impingement

- subscapularis tear

- roller-wringer effect

- anterior shoulder pain

- subcoracoid stenosis

- coracoplasty

Background The incidence of subscapularis tears is increasing as diagnostic imaging and arthroscopic technology improves. Decreased coracohumeral distance (CHD) with associated Subcoracoid Impingement is thought to be one, potential etiology for these lesions.

Objective The purpose of this study was to identify the association between reduced CHD and degenerative pathology of the subscapularis tendon.

Methods A comparative case-control study was performed. The sample size required to determine significance was calculated to be 36 cases. In total, 46 patients with severe degenerative SSC tendinopathy or subscapularis tears on magnetic resonance imaging (MR) were collected consecutively from our database (SSC group). The control group consisted of 36 asymptomatic volunteers undergoing shoulder MR. Only patients between 40 and 60 years of age were included. An independent $t$-test was used to determine the statistical significance between the two groups (with a $95 \%$ level of confidence).

Results No statistically relevant difference was found between the average age of the two groups $(50.1 \pm 6.1$ and $51.7 \pm 6.8 ; p=0.43)$. In the control group, the mean CHD was found to be $11.04 \mathrm{~mm}$ [Cl:10.05-12.04 mm] and was $8.58 \mathrm{~mm}$ [Cl: 7.95-9.21 $\mathrm{mm}]$ in the subscapularis group. The statistical analysis, comparing the CHD showed a significant difference between groups $(p=0.00048)$.

Conclusion This study supports the conclusion that degenerative subscapularis pathology is associated with narrowed coracohumeral distance, when compared with an asymptomatic age-matched group.

Level of evidence III (case-control study).
\end{abstract}

Conclusión Nuestros resultados respaldan la existencia de una asociación estadísticamente significativa entre una DCH disminuida y la presencia de patología degenerativa del SSC.

Nivel de Evidencia: III (Estudio de casos y controles).

\section{Introducción}

Las lesiones degenerativas del tendón del subescapular son una fuente conocida de dolor y disfunción del hombro, ${ }^{1-4}$ que hasta ahora han sido relativamente subestimadas y subdiagnosticadas. ${ }^{5,6}$ Sin embargo, con el advenimiento de nuevos métodos de evaluación y diagnóstico, como la resonancia nuclear magnética y la artroscopía, nuestro conocimiento e interés sobre la real prevalencia de esas lesiones se ha incrementado significativamente en los últimos años. ${ }^{7-13}$

Como resultado de ese fenómeno, varios autores han reportado recientemente que la prevalencia de lesiones del subescapular es más alta de lo que previamente se había publicado, ${ }^{14-20}$ alcanzando, según Lafosse y cols., hasta un $49 \%$ cuando se consideran dentro del total de artroscopías realizadas para reparación del manguito rotador. ${ }^{21}$ Esto ha ido de la mano de un aumento exponencial en el número de publicaciones con respecto a distintas técnicas quirúrgicas de reparación y sus resultados, ${ }^{20-46}$ desde la técnica abierta publicada por Gerber en $1991^{22}$ hasta a las diversas técnicas artroscópicas actuales, cuyo primer reporte fue realizado por Burkhart el 2002. ${ }^{25}$ A pesar de eso, a la fecha poco sabemos en detalle sobre los mecanismos etiopatogénicos que originan esas lesiones. En ese sentido, se ha propuesto una asociación entre una distancia coracohumeral disminuida y un aumento de la prevalencia de lesiones degenerativas del tendón del Subescapular, sin embargo, esa teoría es aún controversial.

Es por esto que, el objetivo de nuestro trabajo, consistió en determinar si existe una diferencia estadísticamente significativa de la DCH medida por RM, entre un grupo de pacientes con patología degenerativa del SSCy un grupo control.

\section{Material y Métodos}

Con aprobación del comité de ética de nuestro hospital se diseñó un estudio comparativo de casos y controles, con las siguientes características:

a) Tamaño muestral: se estableció un tamaño muestral mínimo necesario de 36 casos por grupo para detectar una diferencia mínima de $1 \mathrm{~mm}$ entre 2 medias independientes. Para ese cálculo se utilizó el software de libre acceso en internet GRANMO v.7.12, considerando un riesgo alfa de 0,05, $\beta$ de 0,2 y una desviación estándar común de $1,5 \mathrm{~mm}$ en base a lo publicado por Richards y Burkhart. $^{47}$ 
b) Conformación de los Grupos:

- Casos (Grupo SSC): De la base de datos imagenológicos de nuestro hospital (Impax 6.4; Agfa, Greenville, SC, EE. UU.), se rescataron durante un periodo de 6 meses un total de 46 RM de hombro que presentaron tendinopatía degenerativa avanzada $\mathrm{y} / \mathrm{o}$ roturas del tendón del SSC, $8,48-50$ independiente del grado de compromiso asociado del resto del manguito rotador. La decisión de incluir tendinopatías avanzadas fue para controlar en parte, el efecto teórico de una reducción de la DCH secundaria a roturas del SSC según lo descrito por algunos autores. ${ }^{48,51-55}$

- Grupo Control: Durante el periodo de estudio se realizaron en forma paralela $36 \mathrm{RM}$ de hombro a voluntarios asintomáticos sin patología conocida de hombros que aceptaron participar del estudio.

c) Criterios de inclusión y exclusión: Fueron incluidos en ambos grupos solamente pacientes entre 40 y 60 años. Para eliminar la edad como una tercera variable (de confusión), dado que constituye un factor de riesgo independiente que se correlaciona en forma directa con la presencia de roturas del mango rotador. ${ }^{56}$ Por otra parte fueron excluidos todos aquellos pacientes con antecedente traumático antiguo o reciente del hombro. En el Grupo SSC se excluyeron las RM con patología estructural que no fuera del manguito rotador y en el Grupo Control todas las RM con cualquier patología estructural incluyendo el manguito rotador.

d) RM: Todas las RM de hombro fueron realizadas mediante un protocolo estándar definido por el departamento de imagenología de nuestro hospital, realizando secuencias convencionales en un resonador de 1,5-T (Achieva, Philips Medical Systems, Best, Holanda), con el hombro en posición neutra, esto es, con el brazo y antebrazo a un costado del cuerpo y con el pulgar hacia arriba. Todas las RM de ambos grupos fueron analizadas por un radiólogo musculoesquelético.

e) Distancia Coracohumeral (DCH): La medición de la DCH se realizó en forma estándar como está publicado en la literatura, ${ }^{57-59}$ definiéndose para este estudio en particular como la menor distancia posible en milímetros entre la cortical interna de la punta de la coracoides y la cortical anterointerna de la tuberosidad menor del húmero proximal, medida en los cortes axiales de RM (-Fig. 1).

f) Análisis estadístico: Los resultados obtenidos en ambos grupos fueron registrados como promedio más desviación estándar en una hoja de cálculo del programa Excel (Microsoft, Redmont, WA, US.). Para el análisis estadístico se utilizó el software SPSS (versión 11.0, Chicago, IL, US.), seleccionando la prueba $t$ - Student para comparación de muestras independientes, considerando como significativo un $p<0,05$.

\section{Resultados}

En el grupo SSC la edad promedio fue de 51,7 $\pm 6,8$ años, con un $53,3 \%$ de los casos de sexo masculino (- Tabla 1). La DCH promedio encontrada en este grupo fue de $8,58 \mathrm{~mm}$ [IC: $7,95-$ $9,21 \mathrm{~mm}]$. En el grupo Control entretanto, la edad promedio fue $50,1 \pm 6,1$ años, con un 54,5\% de los casos de sexo masculino y una DCH promedio de 11,04 mm [IC: 10,05-12,04 mm]. Al comparar la edad promedio de ambos grupos, no hubo una diferencia estadísticamente significativa $(p=0,43)$. Sin embargo, en relación a la $\mathrm{DCH}$, se encontró una diferencia estadísticamente significativa ( $p=0,00048)$. (-Fig. 2 ).
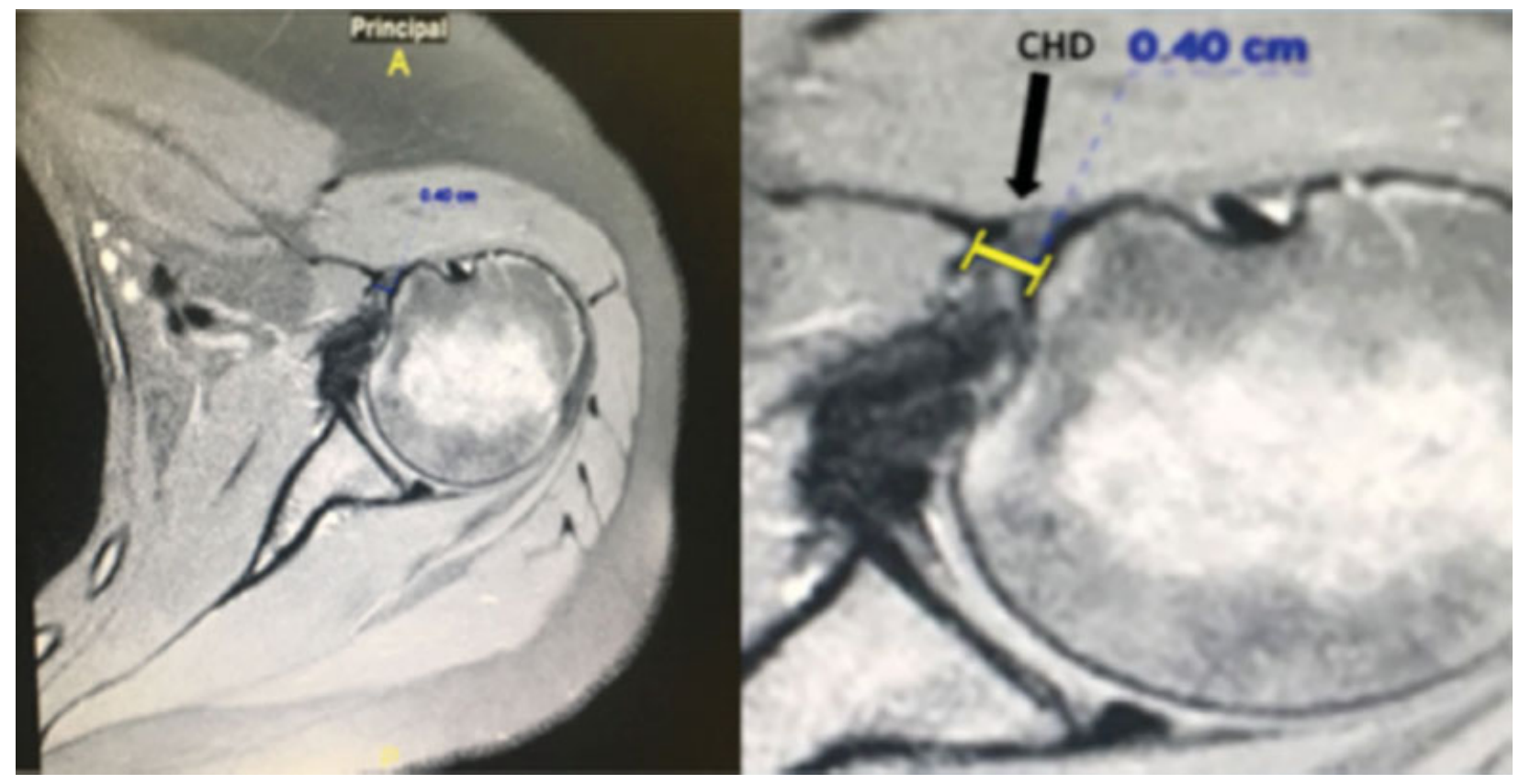

Fig. 1 Medición de la DCH en cortes axiales de RNM. 
Tabla 1 Resultados por grupo

\begin{tabular}{|l|l|l|l|}
\hline & Grupo A (SSC) & Grupo B (control) & $P$ \\
\hline Casos & 46 & 36 & \\
\hline $\begin{array}{l}\text { Edad } \\
\text { (años) }\end{array}$ & $51,7 \pm 6,8$ & $50,1 \pm 6,1$ & 0,43 \\
\hline $\begin{array}{l}\text { Derecho/ } \\
\text { izquierdo }\end{array}$ & $24 / 22$ & $22 / 14$ & \\
\hline $\begin{array}{l}\text { CHD } \\
\text { (mm) }\end{array}$ & $\begin{array}{l}8,58 \pm 2,1 \\
\text { [IC: } 7,95-2,1 \mathrm{~mm}]\end{array}$ & $\begin{array}{l}11,04 \pm 1,48 \\
{[\mathrm{IC}: 10,05-12,04 \mathrm{~mm}]}\end{array}$ & 0,00048 \\
\hline
\end{tabular}

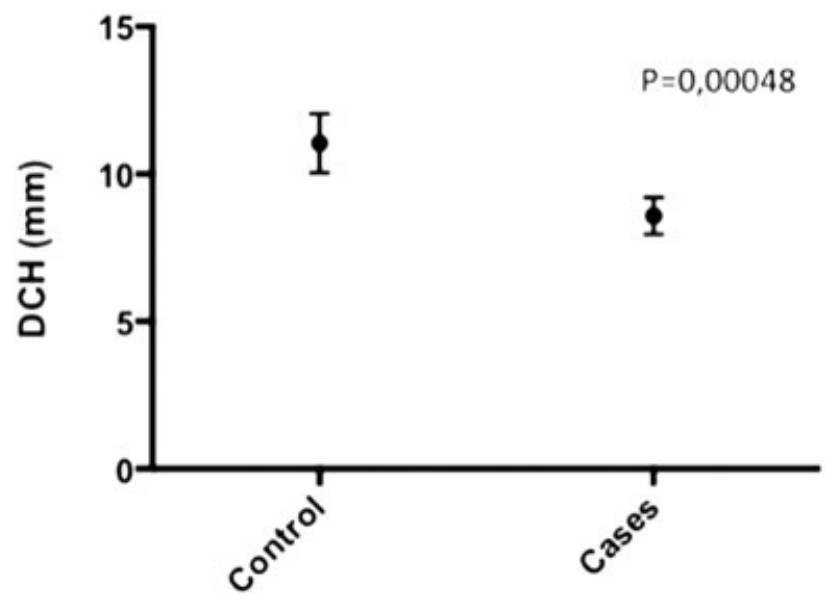

Fig. 2 DCH comparitiva entre grupos (media+ IC 95\%).

\section{Discusión}

Hasta ahora, no existe un claro consenso sobre la etiopatogenia de las lesiones degenerativas del tendón del Subescapular. Una aproximación inicial fue realizada en 1909 por Goldthwait, ${ }^{1}$ quien describió por primera vez un mecanismo que denominó pinzamiento subcoracoideo de la región anterior del hombro. Posteriormente, Gerber y cols., ${ }^{57,60}$ asociaron en forma experimental ese pinzamiento subcoracoideo a la presencia de una disminución anatómica y/o funcional de la $\mathrm{DCH}$, describiendo 3 causas principales de esa verdadera "estenosis subcoracoidea," las idiopáticas, postraumáticas o iatrogénicas.

Sin embargo, fueron Richards y Burkhart ${ }^{47}$ que en el año 2005 publicaron una asociación significativa y clínicamente relevante entre una DCH disminuida y la presencia de lesiones del SSC. Este estudio comparó un grupo de pacientes operados por patología del manguito rotador que requirieron reparación artroscópica del SSC, con una edad promedio de 61,9 años y un grupo control de pacientes también operados por vía artroscópica, pero por una causa distinta al manguito rotador, con una edad promedio de 36,9 años. Ellos encontraron una diferencia estadísticamente significativa entre la DCH de ambos grupos $(5,0 \pm 1,7$ versus $10,0 \pm 1,33$ respectivamente; $p=0,0001)$. No obstante, pese a ese relevante hallazgo, la significativa diferencia en la edad promedio de ambos grupos dificulta las conclusiones, dado que sabemos que en la medida en que aumenta la edad, no solamente aumenta la probabilidad de presentar roturas degenerativas del manguito rotador, ${ }^{56}$ sino que también disminuye progresivamente la $\mathrm{DCH} .{ }^{61}$
Es en ese sentido, que nuestros resultados apoyan una asociación entre DCH y patología degenerativa del SSC, en forma independiente de la edad, ya que encontramos una disminución significativa de la DCH en el grupo SSC cuando lo comparamos con un grupo control sin patología estructural del hombro por RM ( 8,58 vs 11,$04 ; p=0,00048)$, pero sin existir una diferencia significativa entre la edad promedio de ambos grupos ( 51,7 vs 50,$1 ; p=0,43$ ).

Esos resultados son similares a los obtenidos por Aktas y cols., ${ }^{56}$ quienes reportaron una DCH significativamente disminuida por RM en pacientes con cualquier rotura del manguito rotador, al compararlos con un grupo de pacientes $\sin$ rotura $(8,362 \pm 2,382$ versus $9,351 \pm 2,520 ; p=0,031)$.

Apoyando esa misma línea de estudio, recientemente Balke y cols., ${ }^{62}$ compararon la DCH en pacientes con roturas degenerativas, roturas traumáticas y sin roturas del SSC. Ellos encontraron una disminución significativa de la DCH en aquellos pacientes con roturas degenerativas del SSC, al compararlo con el grupo traumático y control, sin existir diferencia significativa entre estos 2 últimos grupos. Los hallazgos los hallazgos antes mencionados constituyen constituyen otro argumento a favor de que una disminución de la DCH se asocia de manera significativa a las roturas degenerativas del SSC.

El mecanismo que explicaría esa asociación fue descrito por Lo y Burkhart, ${ }^{63}$ quienes plantearon que una disminución de la DCH o estenosis subcoracoidea, provocaría un pinzamiento subcoracoideo secundario, ${ }^{64-67}$ que progresivamente conduce a una rotura por sobre tensión de las fibras más profundas o articulares del tendón del SSC en primer lugar (TUFF, tensile undersurface fiber failure), por un fenómeno que denominaron "roller wringer effect" y que finalmente progresa hacia a una rotura completa desde cefálico a caudal.

Sin embargo, más allá de esa teoría, aún no se ha logrado establecer adecuadamente en la literatura una real relación causa-efecto entre DCH y lesiones del SSC. Es más, algunos autores como Patte, ${ }^{51}$ entre otros, ${ }^{48,52-55}$ han señalado que la disminución de la DCH y el pinzamiento subcoracoideo no serían la causa del problema, sino la consecuencia de una inestabilidad glenohumeral anterosuperior secundaria a una rotura masiva del manguito rotador, lo que permite inicialmente una migración anterior dinámica de la cabeza humeral, pero que depués eventualmente terminaría en una subluxación anterior estática.

Por otra parte, las implicancias terapéuticas de esos hallazgos requieren requieren aun mayor estudio al respecto. En ese sentido, la Coracoplastía, por vía abierta ${ }^{52,68-70}$ o artroscópica, ${ }^{71-73}$ ha sido propuesta como un procedimiento para corregir la DCH en pacientes con estenosis subcoracoidea sintomática. Esta técnica, asociada o no a una reparación del tendón del SSC, ha mostrado resultados clínicos satisfactorios, aunque en pequeñas series de casos (Nivel de evidencia tipo IV). ${ }^{27,52,60,68-74}$ Burkhart ha recomendado asociar una coracoplastía artroscópica luego de reparaciones del SSC cuando la DCH sea de $6 \mathrm{~mm}$ o menos, para aumentar el espacio subcoracoideo y con ello disminuir el riesgo de reroturas del SSC. ${ }^{75}$ Sin embargo, en 2 estudios recientes con grupo control, Park $^{76}$ y Kim, ${ }^{77}$ no lograron demostrar la utilidad clínica ni una menor tasa de rerotura del SSC al 
agregar una coracoplastía al momento de la reparación del SSC (Nivel de evidencia tipo III), ${ }^{76,77}$ un fenómeno homologable a la evolución en el tiempo de la acromioplastía en las reparaciones del Supraespinoso. ${ }^{78,79}$

En base a esas publicaciones, y en realidad por falta de ellas, es que por el momento nuestra conducta es expectante con respecto a la Coracoplastía, la cual reservamos solamente para casos seleccionados de pacientes con dolor anterior de hombro y pruebas de pinzamiento subcoracoideo positivas en la evaluación clínica, quienes presentan una rotura degenerativa del SSC por RM, en el contexto de una rotura anterosuperior del manguito rotador, y que además cursan con una estenosis subcoracoidea crítica $(\leq 6 \mathrm{~mm})$ bajo maniobras dinámicas durante la artroscopía. En ese grupo de pacientes asociamos una Coracoplastía por vía extra articular a través de un portal anterolateral una vez abierto el intervalo de los rotadores. Sin embargo, a la fecha no contamos con la experiencia clínica suficiente que nos permita validar nuestra conducta, y es por lo que creemos indispensable continuar realizando nuevos estudios con una mejor calidad metodológica de lo que actualmente disponemos, para comprender mejor tanto el origen como el tratamiento de las lesiones degenerativas del SSC, especialmente en relación a la necesidad de agregar una Coracoplastía en el contexto de una DCH disminuida.

Finalmente, nuestro estudio tiene algunas limitaciones, como por ejemplo la ausencia de correlación clínica y/o artroscópica de los casos estudiados y de los hallazgos obtenidos, así como tampoco cuenta con un estudio preliminar de correlación intra e inter observador que permita validar las mediciones realizadas. Sin embargo, creemos que la incorporación de un radiólogo con experiencia en el área, así como la adecuada estandarización de las mediciones, compensan en parte esa limitación. La selección arbitraria por edad puede constituir de igual manera un sesgo de selección, pero que consideramos razonable y necesaria para evitar una diferencia significativa de la edad entre grupos, como ya fue descrito previamente. Por cierto que también es un estudio con una población pequeña y con algún grado de heterogeneidad en el grupo de estudio, dado que se incluyeron pacientes con estados de pre rotura del SSC (tendinopatías avanzadas), cuya justificación ya fue aclarada en material y métodos.

A pesar de lo anterior, nuestro trabajo tiene algunas fortalezas que vale la pena mencionar, como ser en nuestro conocimiento el primer estudio que define un valor de referencia normal de la $\mathrm{DCH}$ en población nacional $(11,04 \pm 1,48)$, valor que está en plena concordancia con lo publicado en otros estudios internacionales $47,51,57-60,65,66,68$. Sin embargo, a diferencia de esas publicaciones, nuestro estudio contó con un "verdadero" grupo control, es decir, obtenido de voluntarios asintomáticos sin ninguna patología estructural de hombro sometidos a RM, lo cual creemos agrega un valor a los resultados obtenidos.

\section{Conclusión}

Nuestros resultados respaldan una asociación estadísticamente significativa entre una DCH disminuida y la presencia de patología degenerativa del tendón SSC, al compararlo con un grupo control asintomático controlado por edad.

Conflictos de interés

Ninguno

\section{Agradecimientos}

Un especial agradecimiento a todos los voluntarios que colaboraron desinteresadamente en este trabajo, así como también, al equipo de tecnólogos médicos de nuestro hospital, a Susana Baez, Claudio Cortes y Carla Manriquez, quienes facilitaron la adecuada obtención, recopilación y análisis de las imágenes necesarias para este estudio.

\section{Bibliografía}

1 Goldthwait JE. An anatomic and mechanical study of the shoulder joint, explaining many of the cases of painful shoulder, many of the recurrent dislocations and many of the cases of brachial neuralgias or neuritis. J Bone Joint Surg 1909;2-6(04):579-606

2 Inman VT, Saunders JB, Abbott LC. Observations on the function of the shoulder joint. J Bone Joint Surg Am 1944;26(01):1-30

3 Saha AK. Dynamic stability of the glenohumeral joint. Acta Orthop Scand 1971;42(06):491-505

4 Lyons RP, Green A. Subscapularis tendon tears. J Am Acad Orthop Surg 2005;13(05):353-363

5 Lo IK, Burkhart SS. Subscapularis tears: Arthroscopic repair of the forgotten rotator cuff tendon. Tech Shoulder Elbow Surg 2002;3 (04):282-291

6 Lee J, Shukla D, Sánchez-Sotelo J. Subscapularis tears: hidden and forgotten no more.JSES 2018:74-83. Doi: 10.1016/j.jses.2017.11.006

7 Friedman RJ, Bonutti PM, Genez B. Cine magnetic resonance imaging of the subcoracoid region. Orthopedics 1998;21(05):545-548

8 Pfirrmann CW, Zanetti M, Weishaupt D, Gerber C, Hodler J. Subscapularis tendon tears: detection and grading at MR arthrography. Radiology 1999;213(03):709-714

9 Li X-X, Schweitzer ME, Bifano JA, Lerman J, Manton GL, El-Noueam KI. MR evaluation of subscapularis tears. J Comput Assist Tomogr 1999;23(05):713-717

10 Morag Y, Jamadar DA, Miller B, Dong Q Jacobson JA. The subscapularis: anatomy, injury, and imaging. Skeletal Radiol 2011;40(03):255-269

11 Adams CR, Schoolfield JD, Burkhart SS. Accuracy of preoperative magnetic resonance imaging in predicting a subscapularis tendon tear based on arthroscopy. Arthroscopy 2010;26(11):1427-1433

12 Adams CR, Brady PC, Koo SS, et al. A systematic approach for diagnosing subscapularis tendon tears with preoperative magnetic resonance imaging scans. Arthroscopy 2012;28(11): 1592-1600. Doi: 10.1016/j.arthro.2012.04.142

13 Foad A, Wijdicks CA. The accuracy of magnetic resonance imaging and magnetic resonance arthrogram versus arthroscopy in the diagnosis of subscapularis tendon injury. Arthroscopy 2012;28 (05):636-641. Doi: 10.1016/j.arthro.2011.10.006

14 Codman EA. The shoulder. 2nd edition. Boston: Thomas Todd Co.; 1934

15 Frankle MA, Cofield RH. Rotator cuff tears including the subscapularis. In: Proceedings of the Fifth International Conference on Surgery of the Shoulder. Paris: International Shoulder and Elbow Society. Rosemont (IL): American Academy of Orthopaedic Surgeons; 1992

16 Deutsch A, Altchek DW, Veltri DM, Potter HG, Warren RF. Traumatic tears of the subscapularis tendon. Clinical diagnosis, magnetic resonance imaging findings, and operative treatment. Am J Sports Med 1997;25(01):13-22 
17 Sakurai G, Ozaki J, Tomita Y, Kondo T, Tamai S. Incomplete tears of the subscapularis tendon associated with tears of the supraspinatus tendon: cadaveric and clinical studies. J Shoulder Elbow Surg 1998; 7(05):510-515

18 Bennett WF. Subscapularis, medial, and lateral head coracohumeral ligament insertion anatomy. Arthroscopic appearance and incidence of "hidden" rotator interval lesions. Arthroscopy 2001;17(02):173-180

19 Garavaglia G, Ufenast H, Taverna E. The frequency of subscapularis tears in arthroscopic rotator cuff repairs: A retrospective study comparing magnetic resonance imaging and arthroscopic findings. Int J Shoulder Surg 2011;5(04):90-94

20 YooJC, Rhee YG, Shin SJ, et al. Subscapularis tendon tear classification based on 3-dimensional anatomic footprint: a cadaveric and prospective clinical observational study. Arthroscopy 2015;31 (01):19-28

21 Lafosse L, Jost B, Reiland Y, Audebert S, Toussaint B, Gobezie R. Structural integrity and clinical outcomes after arthroscopic repair of isolated subscapularis tears. J Bone Joint Surg Am 2007;89(06):1184-1193. Doi: 10.2106/JBJS.F.00305

22 Gerber C, Krushell RJ. Isolated rupture of the tendon of the subscapularis muscle. Clinical features in 16 cases. J Bone Joint Surg Br 1991;73(03):389-394

23 Gerber C, Hersche O, Farron A. Isolated rupture of the subscapularis tendon. J Bone Joint Surg Am 1996;78(07):1015-1023

24 Warner JJ, Allen AA, Gerber C. Diagnosis and management of subscapularis tears. Tech Orthop 1994;9(2):116-125 . Tech Orthop 1994;9(02):116-125

25 Burkhart SS, Tehrany AM. Arthroscopic subscapularis tendon repair: Technique and preliminary results. Arthroscopy 2002; 18(05):454-463

26 Lo IK, Burkhart SS. The comma sign: An arthroscopic guide to the torn subscapularis tendon. Arthroscopy 2003;19(03):334-337. Doi: $10.1053 /$ jars.2003.50080

27 Lo IK, Parten PM, Burkhart SS. Combined subcoracoid and subacromial impingement in association with anterosuperior rotator cuff tears: An arthroscopic approach. Arthroscopy 2003; 19(10):1068-1078. Doi: 10.1016/ j.arthro.2003.10.016

28 Richards DP, Burkhart SS, Lo IK. Subscapularis tears: arthroscopic repair techniques. Orthop Clin North Am 2003;34(04):485-498

29 Burkhart SS, Brady PC. Arthroscopic subscapularis repair: surgical tips and pearls A to Z. Arthroscopy 2006;22(09):1014-1027

30 Adams CR, Schoolfield JD, Burkhart SS. The results of arthroscopic subscapularis tendon repairs. Arthroscopy 2008;24(12):1381-1389

31 Ticker JB, Burkhart SS. Why repair the subscapularis? A logical rationale. Arthroscopy 2011;27(08):1123-1128. Doi: 10.1016/j. arthro.2011.03.001

32 Denard PJ, Lädermann A, Burkhart SS. Double-row fixation of upper subscapularis tears with a single suture anchor. Arthroscopy 2011;27(08):1142-1149

33 Denard PJ, Lädermann A, Burkhart SS. Arthroscopic management of subscapularis tears. Sports Med Arthrosc Rev 2011;19(04):333-341

34 Lafosse L, Lanz U, Saintmard B, Campens C. Arthroscopic repair of subscapularis tear: Surgical technique and results. Orthop Traumatol Surg Res 2010;96(8, Suppl):S99-S108

35 Edwards TB, Walch G, Sirveaux F, et al. Repair of tears of the subscapularis. J Bone Joint Surg Am 2005;87(04):725-730

36 Edwards TB, Walch G, Nové-Josserand L, et al. Arthroscopic debridement in the treatment of patients with isolated tears of the subscapularis. Arthroscopy 2006;22(09):941-946

37 Bartl C, Scheibel M, Magosch P, Lichtenberg S, Habermeyer P. Open repair of isolated traumatic subscapularis tendon tears. Am J Sports Med 2011;39(03):490-496

38 Bennett WF. Arthroscopic repair of isolated subscapularis tears: A prospective cohort with 2- to 4-year follow-up. Arthroscopy 2003;19(02):131-143

39 Bennett WF. Arthroscopic repair of anterosuperior (supraspinatus/ subscapularis) rotator cuff tears: a prospective cohort with 2- to 4-year follow-up. Classification of biceps subluxation/instability. Arthroscopy 2003;19(01):21-33. Doi: 10.1053/jars.2003.50023

40 Bennett WF. Arthroscopic repair of massive rotator cuff tears: a prospective cohort with 2- to 4-year follow-up. Arthroscopy 2003;19(04):380-390. Doi: 10.1053/jars.2003.50131

41 Kim SH, Oh I, Park JS, Shin SK, Jeong WK. Intra-articular repair of an isolated partial articular-surface tear of the subscapularis tendon. Am J Sports Med 2005;33(12):1825-1830

42 Lucas RM, Iyengar JJ, Ma CB, Feeley BT. Management of subscapularis tears. Tech Shoulder Elbow Surg 2012;13(02): 90-100. Doi: 10.1097/BTE.0b013e31824d61b5

43 Bartl C, Salzmann GM, Seppel G, et al. Subscapularis function and structural integrity after arthroscopic repair of isolated subscapularis tears. Am J Sports Med 2011;39(06):1255-1262

44 Nové-Josserand L, Hardy MB, Leandro Nunes Ogassawara $R$, Carrillon Y, Godenèche A. Clinical and structural results of arthroscopic repair of isolated subscapularis tear. J Bone Joint Surg Am 2012;94(17):e125 [1-6]

45 Toussaint B, Audebert S, Barth J, et al; French Arthroscopy Society (SFA). Arthroscopic repair of subscapularis tears: preliminary data from a prospective multicentre study. Orthop Traumatol Surg Res 2012;98(8, Suppl)S193-S200. Doi: 10.1016/j.otsr.2012.10.004

46 Rhee YG, Lee YS, Park YB, Kim JY, Han KJ, Yoo JC. The outcomes and affecting factors after arthroscopic isolated subscapularis tendon repair. J Shoulder Elbow Surg 2017;26(12):2143-2151

47 Richards DP, Burkhart SS, Campbell SE. Relation between narrowed coracohumeral distance and subscapularis tears. Arthroscopy 2005;21(10):1223-1228

48 Bergin D, Parker L, Zoga A, Morrison W. Abnormalities on MRI of the subscapularis tendon in the presence of a full-thickness supraspinatus tendon tear. AJR Am J Roentgenol 2006;186(02): 454-459. Doi: 10.2214/AJR.04.1723

49 Sein ML, Walton J, Linklater J, et al. Reliability of MRI assessment of supraspinatus tendinopathy. Br J Sports Med 2007;41(08):e9. Doi: 10.1136/bjsm.2006.034421

50 Morag Y, Jacobson JA, Miller B, De Maeseneer M, Girish G, Jamadar D. MR imaging of rotator cuff injury: what the clinician needs to know. Radiographics 2006;26(04):1045-1065

51 Patte D. The subcoracoid impingement. Clin Orthop Relat Res 1990;(254):55-59

52 Dumontier C, Sautet A, Gagey O, Apoil A. Rotator interval lesions and their relation to coracoid impingement syndrome. J Shoulder Elbow Surg 1999;8(02):130-135

53 Nové-Josserand L, Edwards TB, O'Connor DP, Walch G. The acromiohumeral and coracohumeral intervals are abnormal in rotator cuff tears with muscular fatty degeneration. Clin Orthop Relat Res 2005;(433):90-96

54 MacMahon PJ, Taylor DH, Duke D, Brennan DD, O’Brien J, Eustace SJ. Contribution of full-thickness supraspinatus tendon tears to acquired subcoracoid impingement. Clin Radiol 2007;62(06):556-563

55 Li X, Fallon J, Egge N, et al. MRI study of associated shoulder pathology in patients with full-thickness subscapularis tendon tears. Orthopedics 2013;36(01):e44-e50

56 Aktas E, Sahin B, Arikan M, et al. MRI analysis of coracohumeral interval width and its relation to rotator cuff tear. Eur J Orthop Surg Traumatol 2015;25(02):281-286

57 Gerber C, Terrier F, Zehnder R, Ganz R. The subcoracoid space. An anatomic study. Clin Orthop Relat Res 1987;(215):132-138

58 Nové-Josserand L, Boulahia A, Levigne C, Noel E, Walch G. [Coracohumeral space and rotator cuff tears]. Rev Chir Orthop Repar Appar Mot 1999;85(07):677-683

59 Tan V, Moore RS Jr, Omarini L, Kneeland JB, Williams GR Jr Iannotti JP. Magnetic resonance imaging analysis of coracoid morphology and its relation to rotator cuff tears. Am J Orthop 2002;31(06):329-333

60 Gerber C, Terrier F, Ganz R. The role of the coracoid process in the chronic impingement syndrome. J Bone Joint Surg Br 1985;67 (05):703-708 
61 Dugarte AJ, Davis RJ, Lynch TS, Schickendantz MS, Farrow LD. Anatomic Study of Subcoracoid Morphology in 418 Shoulders: Potential Implications for Subcoracoid Impingement. Orthop J Sports Med 2017;5(10):2325967117731996

62 Balke M, Banerjee M, Greshake O, Hoeher J, Bouillon B, Liem D. The Coracohumeral Distance in Shoulders With Traumatic and Degenerative Subscapularis Tendon Tears. Am J Sports Med 2016;44(01):198-201. Doi: 10.1177/0363546515611653

63 Lo IKY, Burkhart SS. The etiology and assessment of subscapularis tendon tears: a case for subcoracoid impingement, the rollerwringer effect, and TUFF lesions of the subscapularis. Arthroscopy 2003;19(10):1142-1150. Doi: 10.1016/j.arthro.2003.10.024

64 Giaroli EL, Major NM, Lemley DE, Lee J. Coracohumeral interval imaging in subcoracoid impingement syndrome on MRI. AJR Am J Roentgenol 2006;186(01):242-246

65 Russo R, Togo F. The subcoracoid impingement syndrome: clinical, semeiologic and therapeutic considerations. Ital J Orthop Traumatol 1991;17(03):351-358

66 Ferrick MR. Coracoid impingement. A case report and review of the literature. Am J Sports Med 2000;28(01):117-119

$67 \mathrm{Oh} \mathrm{JH}$, Song BW, Choi JA, Lee GY, Kim SH, Kim DH. Measurement of coracohumeral distance in 3 shoulder positions using dynamic ultrasonography: correlation with subscapularis tear. Arthroscopy 2016;32(08):1502-1508

68 Dines DM, Warren RF, Inglis AE, Pavlov H. The coracoid impingement syndrome. J Bone Joint Surg $\mathrm{Br}$ 1990;72(02): 314-316

69 Suenaga N, Minami A, Kaneda K. Postoperative subcoracoid impingement syndrome in patients with rotator cuff tear. J Shoulder Elbow Surg 2000;9(04):275-278

70 Kragh JF Jr, Doukas WC, Basamania CJ. Primary coracoid impingement syndrome. Am J Orthop 2004;33(05):229-232, discussion 232
71 Karnaugh RD, Sperling JW, Warren RF. Arthroscopic treatment of coracoid impingement. Arthroscopy 2001;17(07):784-787

72 Lo IK, Burkhart SS. Arthroscopic coracoplasty through the rotator interval. Arthroscopy 2003;19(06):667-671. Doi: 10.1016/ S0749-8063(03)00219-6

73 Kleist KD, Freehill MQ Hamilton L, Buss DD, Fritts H. Computed tomography analysis of the coracoid process and anatomic structures of the shoulder after arthroscopic coracoid decompression: a cadaveric study. J Shoulder Elbow Surg 2007;16(02):245-250

74 Garofalo R, Conti M, Massazza G, Cesari E, Vinci E, Castagna A. Subcoracoid impingement syndrome: a painful shoulder condition related to different pathologic factors. Musculoskelet Surg 2011;95(Suppl 1):S25-S29

75 Stephen S. Burkhart, M.D. ICL 406: Biomechanics and Etiology of Tears of the Subscapularis. 2012 AANA Annual Meeting Orlando, Florida, US

76 Park JY, Lhee SH, Oh KS, Kim NR, Hwang JT. Is arthroscopic coracoplasty necessary in subcoracoid impingement syndrome? Arthroscopy 2012;28(12):1766-1775

77 Kim SJ, Choi YR, Jung M, Yoon YK, Chun YM. Concomitant coracoplasty during arthroscopic subscapularis repair does not yield better clinical outcomes and structural integrity. Knee Surg Sports Traumatol Arthrosc 2018;26(01):56-62. Doi: 10.1007/ s00167-016-4368-7

78 MacDonald P, McRae S, Leiter J, Mascarenhas R, Lapner P. Arthroscopic rotator cuff repair with and without acromioplasty in the treatment of full-thickness rotator cuff tears: a multicenter, randomized controlled trial. J Bone Joint Surg Am 2011;93(21): 1953-1960

79 Shin SJ, Oh JH, Chung SW, Song MH. The efficacy of acromioplasty in the arthroscopic repair of small- to medium-sized rotator cuff tears without acromial spur: prospective comparative study. Arthroscopy 2012;28(05):628-635 